\title{
Traité de médecine d'urgence de la personne âgée. Boddaert J, Ray P, Éditions Arnette, Paris, 2011, 1270 pages
}

\author{
B. Riou \\ (C) SFMU et Springer-Verlag France 2011
}

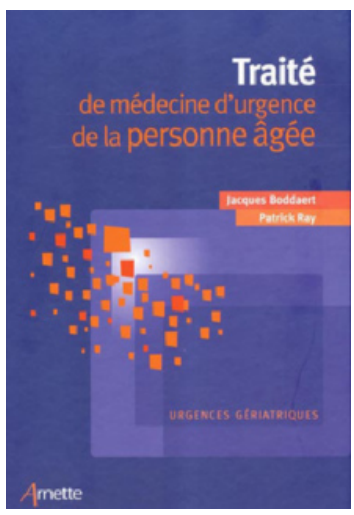

Ce premier traité de médecine d'urgence de la personne âgée est un ouvrage multidisciplinaire tout à fait remarquable qui détaille les grands principes de la médecine d'urgence chez ces patients et l'impact du vieillissement sur son fonctionnement. En s'entourant d'auteurs nationaux de renom, gériatres, urgentistes, mais aussi spécialistes d'organe, Jacques Boddaert et Patrick Ray proposent au lecteur d'approfondir leurs connaissances grâce aux données exhaustives tirées de la littérature la plus récente, mais aussi grâce à leur expérience sur le terrain. Les deux auteurs, le premier gériatre, le second urgentiste, ont réussi à produire un ouvrage de grande qualité qui traduit l'interdisciplinarité nécessaire à la prise en charge de ces patients. Cet ouvrage devrait permettre à la fois au médecin urgentiste d'accéder aux connaissances gériatriques indispensables à son exercice quotidien face à une population vieillissante, et au médecin gériatre de mieux comprendre les enjeux modernes des urgences dans la prise en charge de sa patientèle. Cet ouvrage est constitué de rappels physiopathologiques, comprend de nombreux arbres diagnostiques, avec la description des gestes techniques les plus fréquents, et décrit les outils diagnostiques utilisables en médecine d'urgence. Outre les grands principes de recours et les grandes pathologies vitales et d'urgences " relatives ", les auteurs proposent également une partie entière dédiée à l'utilisation des médicaments et à leur iatrogénie chez la personne âgée. Ce traité devrait avoir un beau succès auprès des gériatres et urgentistes. Il convient de noter que ces mêmes auteurs ont également mis en place un diplôme d'université sur cette thématique à l'université Pierre-et-Marie-Curie-Paris-VI.

Pour tout renseignements, contacter le Pr Jacques Boddaert ; e-mail : jacques.boddaert@psl.aphp.fr

B. Riou

Service d'accueil des urgences, groupe hospitalier Pitié-Salpêtrière, université Pierre-et-Marie-Curie-Paris-VI, Paris, France 